\title{
Evaluation of Iron Deposition in the Adrenal Glands of $\beta$ Thalassemia Major Patients Using 3-Tesla MRI
}

\author{
Tevfik Guzelbey, ${ }^{1}$ Bengi Gurses, ${ }^{2,}$ Erman Ozturk, ${ }^{3}$ Olcay Ozveren, ${ }^{4}$ Aysegul Sarsilmaz, ${ }^{1}$ and Ebru \\ Karasu $^{1}$ \\ ${ }^{1}$ Department of Radiology, School of Medicine, Yeditepe University, Istanbul, Turkey \\ ${ }^{2}$ Department of Radiology, School of Medicine, Koc University, Istanbul, Turkey \\ ${ }^{3}$ Department of Hematology, School of Medicine, Koc University, Istanbul, Turkey \\ ${ }^{4}$ Department of Cardiology, School of Medicine, Yeditepe University, Istanbul, Turkey \\ "Corresponding author: Bengi Gurses, Department of Radiology, School of Medicine, Koc University, Istanbul, Turkey. E-mail: bgurses@kuh.ku.edu.tr
}

Received 2016 January 15; Revised 2016 February 25; Accepted 2016 March 29.

\begin{abstract}
Background: Beta-thalassemia major ( $\beta$-TM) patients need blood transfusions, which result in iron deposition. To regulate chelation therapy, iron load has to be measured. With MRI, the amount of signal loss and T2* decay time shortening are used for iron quantification.

Objectives: The aim was to measure adrenal iron load with T2* relaxometry using MRI, and to compare it with liver and cardiac iron and serum ferritin, and to find out whether adrenal iron could be predicted from those parameters.

Patients and Methods: Between October 2014 and March 2015, MRI was performed in 21 patients with $\beta$-TM, recieving blood transfusions and chelation therapy. The control group $(n=11)$ included healthy volunteers with no known history of adrenal, hematologic, chronic disease, and blood transfusion.

Results: Among patients, there was no significant correlation between plasma ferritin and adrenal T2*. Significant difference was detected among $\mathrm{T}^{*}$ values of adrenals between the patient and control groups. There was no significant correlation between adrenal gland and liver T2* in $\beta$-TM patients, moderate correlation was detected between adrenal T2* and cardiac T2*.

Conclusion: Adrenal iron in $\beta$-TM can be reliably measured in 3 Tesla MRI. The results highlight the absence of correlation between adrenal iron deposition both with serum ferritin and hepatic iron.
\end{abstract}

Keywords: Adrenal Glands, Magnetic Resonance Imaging, Thalassemia, Relaxation

\section{Background}

Beta-thalassemia major ( $\beta$-TM) is a hereditary type of hematological disease characterized by impairment in the production of beta globulin chain of hemoglobin, resulting in chronic severe hemolytic anemia. Autosomal recessively inherited thalassemias are the most common type of single-gene disease worldwide (1). $\beta$-TM patients need regular blood transfusions throughout their life, which in turn results in excessive iron deposition. The excessive iron deposits exhibit toxic effects in various organs causing organ dysfunction, mostly presenting with hepatic, cardiac and endocrine complications $(1,2)$. The most frequently encountered endocrinologic disorders are hypogonadism, diabetes, lack of growth hormone and short stature, primary hypothyroidism, and hypoparathyroidism (3).

It is well known that with the introduction of chelation therapies, substantial improvements have been achieved in both life expectancy, and also the quality of life in $\beta$ TM patients (4). In order to regulate the dosage and frequency of chelation therapy, the iron load has to be mea- sured and monitored, which has been predicted classically by measuring serum ferritin values. Various data have shown that there is good correlation between liver iron load and serum ferritin values (5), whereas no significant correlation has been observed between cardiac iron load and serum ferritin values and liver iron load $(6,7)$. Currently, it has been shown in a few studies that apart from cardiac overload, relying on low serum ferritin levels and liver iron load would be misleading also for the prediction of splenic, pancreatic and total body iron accumulation status (8). Due to the above-mentioned limitations, there is ongoing research for a non-invasive and efficient technique to predict and monitor iron overload in various organ systems. In the last years, in order to evaluate the iron load in the liver and the heart, magnetic resonance imaging (MRI) has been established as an effective non-invasive tool alternative to biopsy (9). Iron with its superparamagnetic nature increases the longitudinal and transverse relaxation rates of protons, with a prononunced affect on transverse relaxation rates that is more pronounced on 
gradient echo sequences. The amounts of signal loss and $\mathrm{T}^{*}$ decay time shortening are used for quantification of iron accumulation in tissues with different techniques.

MRI has currently become a widely clinically accepted method for guidance of chelation treatment in $\beta$-TM patients for evaluation of the liver and heart iron load, with high sensitivity $(9,10)$. However, most of the previous research has been carried out in the liver and heart, and there is limited data about the potential of MRI in the evaluation of iron load in other organs. A few authors have published data regarding the role of MR in the evaluation of iron accumulation in the spleen, pancreas, bone marrow, and pituitary glands $(8,11,12)$.

The deposition of iron in the adrenal glands has been shown histologically in the autopsy series of patients with hemosiderosis (3). Additionally, functional alterations in the adrenal glands due to iron deposition have been described previously in the literature (13). As to our knowledge, only Drakonaki et al. (3) have evaluated iron deposition in the adrenal glands using MRI. They have compared the control and diseased groups according to adrenal gland signal intensity values in a semi-quantitative way, using a three point scale. Their data have shown the presence of significant correlation between iron accumulation in the adrenal gland and the liver, but no significant correlation between adrenal iron load and serum ferritin levels (3).

The vast majority of data regarding iron measurements with MRI in various organs have been carried out with 1.5 Tesla MRI equipments. As 3 Tesla systems have become more widely available for clinical practice, despite the presence of various technical challenges related with iron measurements, we believe that it is necessary now to optimize the parameters and to overcome the technical obstacles for the correct assessment of iron load.

\section{Objectives}

The aim of this study was to measure adrenal gland iron load in $\beta$-TM patients with $\mathrm{T} 2 *$ relaxometry technique in 3 Tesla MRI system, and to compare the results with liver, cardiac iron load and serum ferritin levels, to find out whether adrenal iron accumulation could be predicted from those parameters.

\section{Patients and Methods}

\subsection{Study Population}

Between October 2014 and March 2015, MRI was performed on a total of 21 patients with the diagnosis of $\beta$-TM, recieving regular blood transfusions and chelation therapy to evaluate iron accumulation in the adrenal glands. Two patients were excluded from the study after MRI examination, since one patient had an angiomyolipoma (AML) in the right adrenal gland and the images of the other patient had intense motion artifacts. Among 19 patients included in the study, 11 were female and the remaining were male. The age range of the patient population was 22 46 years (mean age: $29.3 \pm 7.6$ ). All patients recieved systematic red blood cell transfusions at 2 - 4 week intervals. Chelation therapy was performed with oral deferasirox (20 - $40 \mathrm{mg} / \mathrm{kg} /$ day), oral deferipron $(75-100 \mathrm{mg} / \mathrm{kg} /$ day), and/or subcutaneous deferoxamine $(40 \mathrm{mg} / \mathrm{kg} /$ day $)$. The mean duration of blood transfusion was $28.38 \pm 7.63$ years, whereas the mean duration of chelation therapy was 23.9 \pm 3.01 years for the patient group.

All the MRI examinations were performed one week before the blood transfusion. Plasma ferritin levels were measured maximum 2 weeks before blood transfusion. The control group ( $\mathrm{n}=11)$ was selected from healthy volunteers, with no known history of adrenal, hematologic, chronic disease, or blood transfusion, and included individuals without any significant difference compared to the patient group in terms of age and gender. The control group consisted of five female and six male subjects with a mean age of 32.4 (age range: 22 - 41 years). The study was approved by the local ethics committee. All subjects were informed about the procedure and detailed written informed consent was obtained from each individual before the MRI examination.

\subsection{Magnetic Resonance Imaging \\ 3.2.1. Technique}

MRI examinations were performed with a 3 Tesla MRI machine (Ingenia, Philips, Best, The Netherlands) equipped with high-performance gradients with a maximum strength of $80 \mathrm{mT} / \mathrm{m}$ and slew rate of $200 \mathrm{mT} / \mathrm{m} / \mathrm{ms}$, using 16-channel phased-array SENSE-compatible Torso coil. All the MRI examinations were performed in supine position. ECG and respiratory pads were inserted for triggering when needed. As far as breath hold sequences are concerned, respiration was held at the end of expiration in order to achieve standardization and minimize artifacts.

In order to demonstrate, localize and evaluate adrenal glands, initially a three dimensional (3D) multi-echo mDIXON sequence was obtained in the axial plane (TR: 3.9 $\mathrm{ms}$, TE : shortest, flip angle $10^{\circ}$, matrix $488 \times 292$, FOV 400 $\mathrm{mm}$, slice thickness: $2 \mathrm{~mm}$ ) from which in-phase, opposedphase, fat and water images were reconstructed. The acquired images were evaluated in terms of the location, size and presence of any imaging abnormality (e.g. mass lesion, and hyperplasia). To evaluate iron load, fat supressed, 
gradient turbo field echo (TFE) sequence with 16 different echo times in the range of 2.1 - $25 \mathrm{~ms}$ including both adrenal glands and a portion of the liver was obtained in the axial plane (TR: $28 \mathrm{~ms}$, flip angle $20^{\circ}$, matrix: $168 \times$ 320 , FOV $370 \mathrm{~mm}$, slice thickness $5 \mathrm{~mm}$ ). For each subject, a total of 10 slices was obtained for the adrenals in multiecho sequences. For cardiac iron load measurements, breath-hold, ECG-triggered, black-blood, fat supressed TFE sequence (TR: $28 \mathrm{~ms}$, flip angle $20^{\circ}$, matrix: $146 \times 288$, FOV $350 \mathrm{~mm}$, slice thickness $5 \mathrm{~mm}$ ) was obtained in the short axis with 16 different echo times (TE range: 2.1 - $25 \mathrm{~ms}$ ). A single slice was acquired in the short axis plane passing through the mid-ventricular level. The mean duration of the MRI examination was about 45 minutes.

\subsubsection{Image Interpretation and Analysis}

Initially, all MRI images were reviewed to assess the adrenal gland size to exclude any possible hyperplasia and the presence of any mass lesions. The maximum width for each adrenal body and both limbs were measured and recorded. The size of the adrenal glands were considered normal if the width of the body was less than $1 \mathrm{~cm}$, and the limbs were less than $0.42 \mathrm{~cm}$, according to the previously defined criteria on cross-sectional imaging (14).

After morphological evaluation, iron load measurements were performed with relaxometry technique on T2* weighted images, using a special software (CMR tools, cardiovascular imaging solutions, London, UK). Measurements on the adrenal glands were performed separately from both sides. The free-hand regions of interest (ROI) were drawn on the adrenal glands on the body and both limbs separately. Extreme care was taken not to include any pixel from outside the gland (Figure 1). Three separate measurements were performed for each gland, two from the limbs, one from the body, and the mean value was used for statistical analysis. For liver iron load measurements, three separate ROI's, two on the right and one on the left lobe were drawn (Figure 2A). During ROI replacement on the liver, possible artifacts from vessels and respiration were avoided carefully. In patients with high iron load, the measurements were performed from only first three echo times (TE). The cut-off value for liver iron overload was regarded as $3.3 \mathrm{~ms}$, values between 1.4 and $3.3 \mathrm{~ms}$ were accepted as mild, 0.7 and $1.4 \mathrm{~ms}$ as moderate and $<0.7$ $\mathrm{ms}$ as severe iron load, regarding the formula suggested by Storey et al. (15), for adaptation of values from $1.5 \mathrm{~T}$ to $3 \mathrm{~T}$ systems. For cardiac iron measurements, ROI's are placed at the interventricular septum including the endo- and epicardium at the slice obtained in the mid-ventricular level (Figure 2B). Great care was taken for the T2* decay curve to be under the 0.9 of R-square value. The cut-off value for cardiac iron overload was accepted as $12 \mathrm{~ms}$, the range of 7.8-
$12 \mathrm{~ms}$ was classified as mild, 5.4 - $7.8 \mathrm{~ms}$ as moderate and $<5.4 \mathrm{~ms}$ as severe cardiac iron deposition, regarding the formula suggested by Storey et al. (15), for adaptation of values from $1.5 \mathrm{~T}$ to $3 \mathrm{~T}$ systems. All the measurements were performed by a radiologist experienced in body MRI. All the above-mentioned measurements were performed for both the control and the study group.

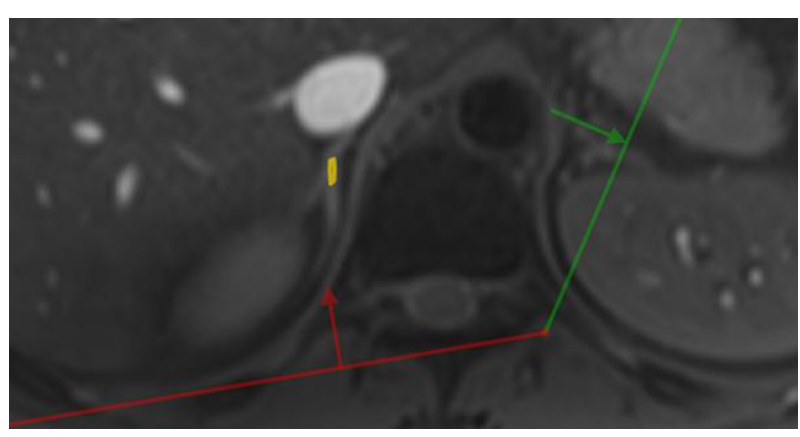

Figure 1. T2* measurement on the medial limb of the adrenal gland with free-hand region of interest (ROI) is shown.

\subsection{Statistical Analysis}

The data were analysed using SPSS ver. 22.0 (IBM Corp., Released 2013, IBM SPSS statistics for windows, version 22.0 Armonk, NY, USA). The convenience of the data to normal distribution was evaluated by Kolmogorov-Smirnov test. The statistical results were designated as mean \pm standard deviation (SD) for continuous variables, and as frequency and percentage for categorical variables. In the presence of two independent groups convenient to normal distribution, for comparison, independent samples-t-test was used. For data that did not exhibit normal distribution, Mann-whitney U test was employed for comparison. In order to analyze whether there is correlation between the two variables, Pearson's correlation coefficient was calculated. P values below 0.05 were regarded as statistically significant.

\section{Results}

Two patients were excluded from the study. In one patient, a $10 \mathrm{~cm}$ mass was observed in the right adrenal gland at the initial m-DIXON sequence, which was evaluated as angiomyolipoma due to characteristic signal properties. In the remaining subjects, the size and morphology of both adrenal glands were in normal limits. No mass lesion, or hyperplasia was observed. Intense motion artifacts were noticed in the other patient and MRI examination was stopped after the initial sequence was obtained. 

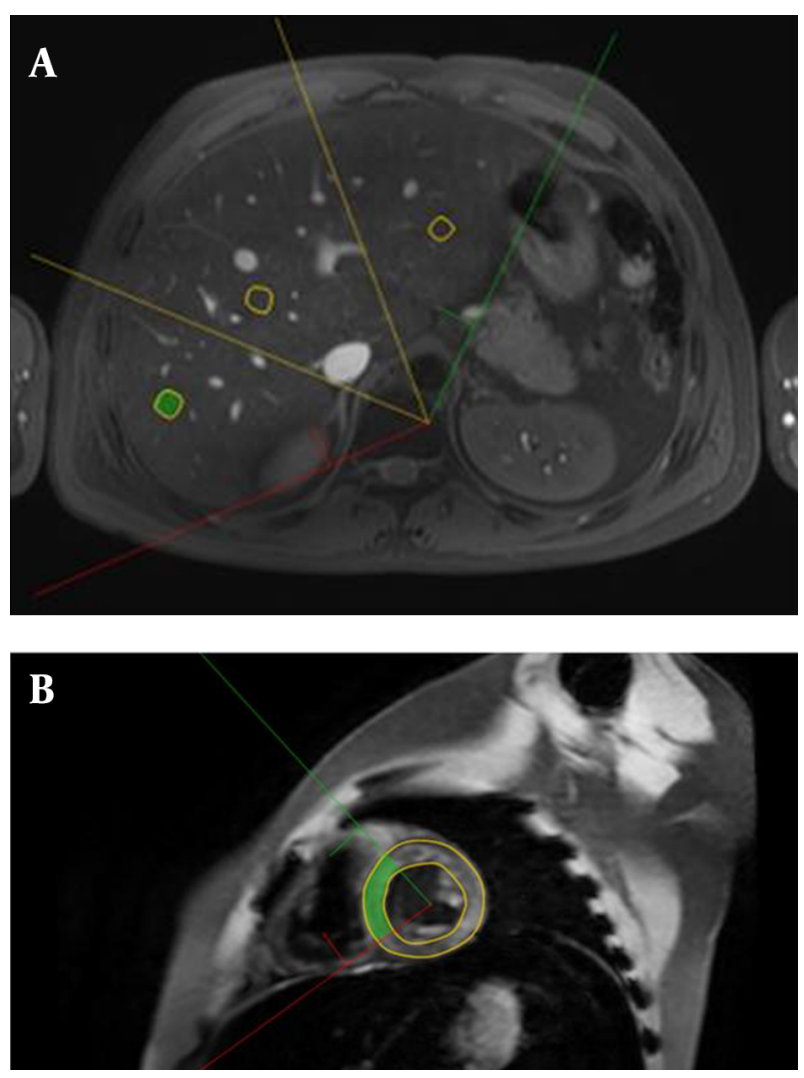

Figure 2. $\mathrm{A}, \mathrm{T} 2^{*}$ measurement of the right lobe of the liver with free-hand ROI is shown; B, T2* measurement of the interventricular septum in a thalassemia patient with severe cardiac iron deposition with free-hand ROI including endo-, myo- and epicardial layers is shown. T2* decay curve is under the 0.9 of R-square value.

The mean $\mathrm{T}^{*}$ values of the liver, heart and adrenal glands in control and patient groups are shown in detail in Table 1 . There was no statistically significant difference for age and gender distribution among the patient and control groups $(\mathrm{P}>0.05)$. There was no statistically significant difference between the mean $\mathrm{T} 2 *$ values of the right and left adrenal glands among all study populations $(24.85 \pm 15.32$ $\mathrm{ms}$, and $24.46 \pm 11.78 \mathrm{~ms}$, respectively) ( $\mathrm{P}>0$ 0 05). There was strong correlation between the $\mathrm{T} 2 *$ values of both sides (Pearson's correlation coefficient, $\mathrm{r}=0.803, \mathrm{P}<0.001$ ). For this reason, in the following statistical analyses and measurements, the mean values of both sides were used.

Serum ferritin levels were measured in the patient group. All the measurements were performed at least two weeks before the blood transfusion. The mean serum ferritin value was calculated as $2255.3 \mathrm{ng} / \mathrm{mL}$ with a range of $442 \mathrm{ng} / \mathrm{mL}$ to $8521 \mathrm{ng} / \mathrm{mL}$ (normal value range 20-300 $\mathrm{ng} / \mathrm{mL}$ ). In each individual of the patient group, the serum ferritin value was higher than normal.

Statistically significant difference was detected among
T2* values of adrenal glands between the patient (mean: $18.78 \pm 11.44 \mathrm{~ms})$ and control $(34.81 \pm 8.74 \mathrm{~ms})$ groups $(\mathrm{P}$ $<0.001$ ) (Figure 3 ). In $68 \%$ of $\beta$-TM patients, the $\mathrm{T}^{*}$ value of adrenals was found to be less than the control group. The cut- off value for liver iron overload was regarded as 3.3 ms, values between 1.4 and $3.3 \mathrm{~ms}$ were accepted as mild, 0.7 and $1.4 \mathrm{~ms}$ as moderate and $<0.7 \mathrm{~ms}$ as severe iron load (15) (Figure 4). According to these criteria, six subjects (31.5\%) in the patient group had no liver iron overload, whereas nine (47.3\%) had mild, two had (10.5\%) moderate, and the remaining two had (10.5\%) severe hepatic iron load. There was no statistically significant correlation between adrenal gland $\mathrm{T} 2 *$ value and liver $\mathrm{T} 2 *$ value in $\beta$ TM patients $(\mathrm{P}>0.05)$, whereas moderate correlation was detected between adrenal $\mathrm{T} 2 *$ and cardiac $\mathrm{T} 2{ }^{*}$ values $(\mathrm{r}=$ $0.684, \mathrm{P}=0.001$ ). There was no significant difference between adrenal $\mathrm{T} 2 *$ values among subjects with liver $\mathrm{T} 2 *$ values $<3.26 \mathrm{~ms}(\mathrm{n}=13)$ and liver T2* values $>3.26 \mathrm{~ms}(\mathrm{n}=5)(P$ $>0.05)$. The cut-off value for cardiac iron overload was accepted as $12 \mathrm{~ms}$, the range of 7.8-12 ms was classified as mild, 5.4 - $7.8 \mathrm{~ms}$ as moderate and $<5.4 \mathrm{~ms}$ as severe cardiac iron deposition (15). In the patient group, 14 subjects (\%73.6) were detected to have cardiac iron values in normal range, two patients (10.5\%) had mild, two patients (10.5\%) moderate and the remaining one patient (\%5.2) had severe cardiac iron deposition. Statistically significant difference was observed between adrenal gland $\mathrm{T} 2 *$ values among subjects with cardiac $\mathrm{T} 2 *$ value $<12 \mathrm{~ms}(\mathrm{n}=5)$ and cardiac $\mathrm{T} 2 *$ value $>12 \mathrm{~ms}(\mathrm{n}=14)(\mathrm{P}<0.001)$. Moderate correlation was detected between adrenal $\mathrm{T} 2 *$ and cardiac $\mathrm{T} 2 *$ values $(\mathrm{r}=$ $0.684, \mathrm{P}=0.001$ ).

Among $\beta$-TM patients, there was no statistically significant difference between plasma ferritin and adrenal gland $\mathrm{T} 2 *$ values $(\mathrm{P}>0.05)$.

\section{Discussion}

In $\beta$-TM one of the most important etiologies of morbidity and mortality is hemosiderosis due to frequent blood transfusions $(1,2)$. Normally, iron is transported by transferrin, and the iron saturation value of transferrin is $20 \%-35 \%$. On the other hand, in case of transfusion related anemias, this value may increase up to $100 \%$. Iron not bound to transferrin (non-transferrin bound iron - NTBI) emerges when the binding capacity of transferrin is exceeded. NTBI is bound to albumin, citrate, amino acids, carbohydrates, and other small molecules loosely and behaves different to normal transferrin bound iron. NTBI has been shown to be taken up by the liver and heart approximately 200 times faster than transferrin bound iron. When the intracellular iron storage capacity of ferritin is 
Table 1. Mean T2* Values of Adrenal Glands, Liver and Cardiac Interventricular Septum in Control and Patient Groups

\begin{tabular}{|c|c|c|c|c|c|}
\hline \multirow[t]{2}{*}{ Mean T2*, ms } & \multicolumn{2}{|c|}{ Control Group } & \multicolumn{2}{|c|}{ Patient Group } & \multirow[t]{2}{*}{ P Value } \\
\hline & Mean \pm SD & Min - Max & Mean \pm SD & Min - Max & \\
\hline Adrenal glands & $34.81 \pm 8.74$ & $24.19-50.91$ & $18.78 \pm 11.44$ & $5.53-50.07$ & 0.001 \\
\hline Liver & $19.72 \pm 2.54$ & $15.67-23.28$ & $3.30 \pm 3.37$ & $0.63-14.92$ & 0.001 \\
\hline Heart & $26.02 \pm 2.40$ & $21.88-30.03$ & $19.39 \pm 10.40$ & $3.18-37.68$ & 0.01 \\
\hline
\end{tabular}

Abbreviations: Min : minimum; Max: maximum; ms: miliseconds; SD: standard deviation
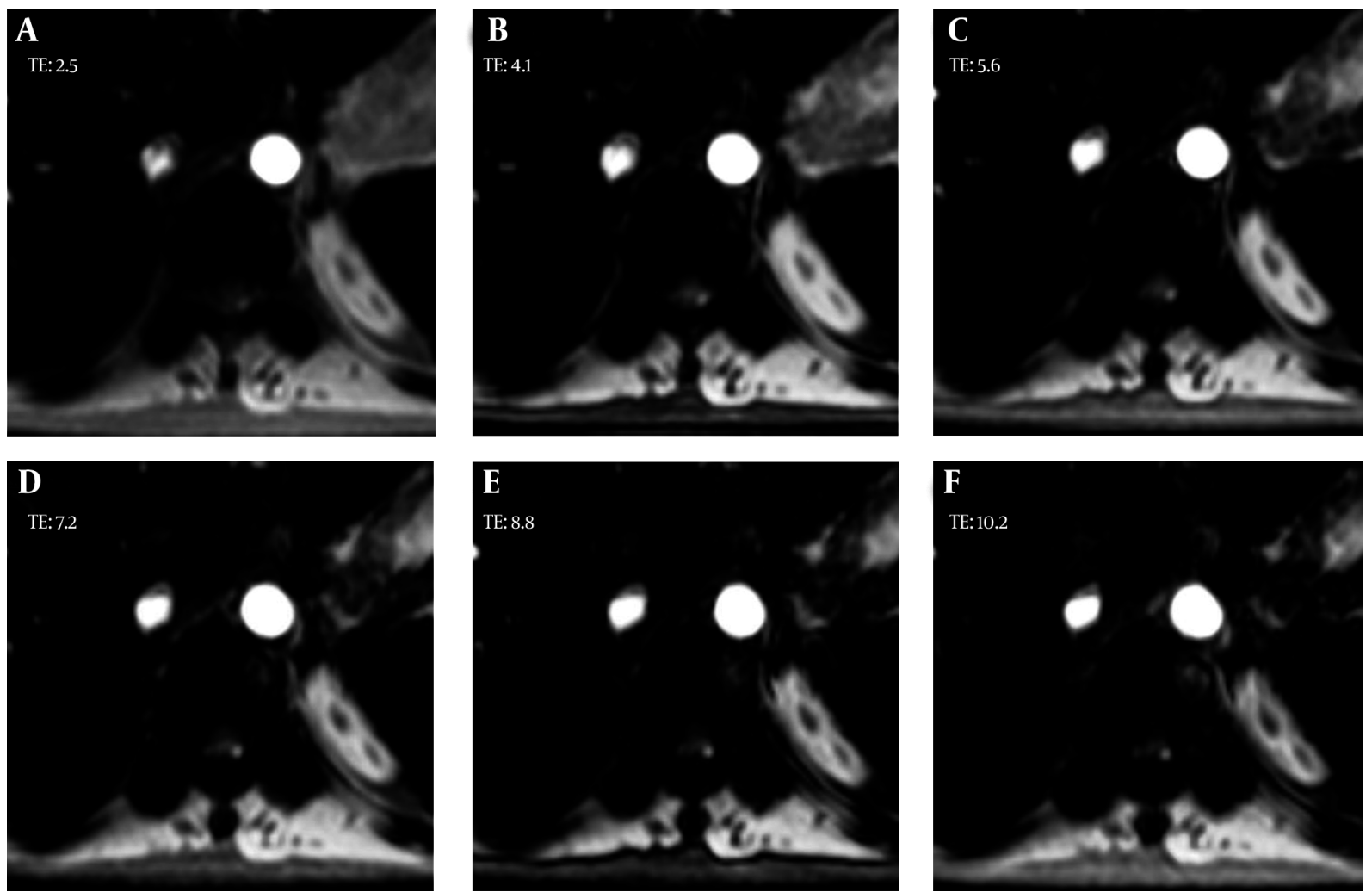

Figure 3. A - F, Multiecho fat supressed, gradient turbo field echo images in the axial plane at gradually increasing echo times passing through the level of the left adrenal gland in a thalassemia patient. As the echo-time increases, there is drammatical signal loss in the left adrenal gland, whose borders become very hard to distinguish after certain echo time values.

exceeded, the labile intracellular iron via the fenton reaction causes formation of free hydroxyl radicals. The hydroxyl radicals may cause damage to the DNA and proteins and also result in peroxidation of lipids and organelle dysfunction $(1,2,4)$. Iron overload is well known to produce damage in various organs and has been described to cause endocrinological function disturbances when accumulated in the hypophysis, thyroid, parathyroid, endocrine pancreas, and adrenals (3).

Iron overload has been demonstrated by imaging, and also histologically $(5,6,16)$. Autopsy studies reveal that iron overload in the adrenal gland preferentially occurs at the zona glomerulosa where mineralocorticoid synthesis takes place. On the other hand, to a lesser extent iron deposition also has been shown in the zona fasciculata where cortisol synthesis occurs. Studies performed on $\beta$ TM patients have shown that the prevalance of biochemical adrenal insufficiency depends on the clinical course of the disease, age of the patient, transfusion load, degree of iron accumulation, and chelation therapy. The prevalence has been reported as 13\% - 61\% although there are variations among diagnostic tests and cut-off values (16-18). In 

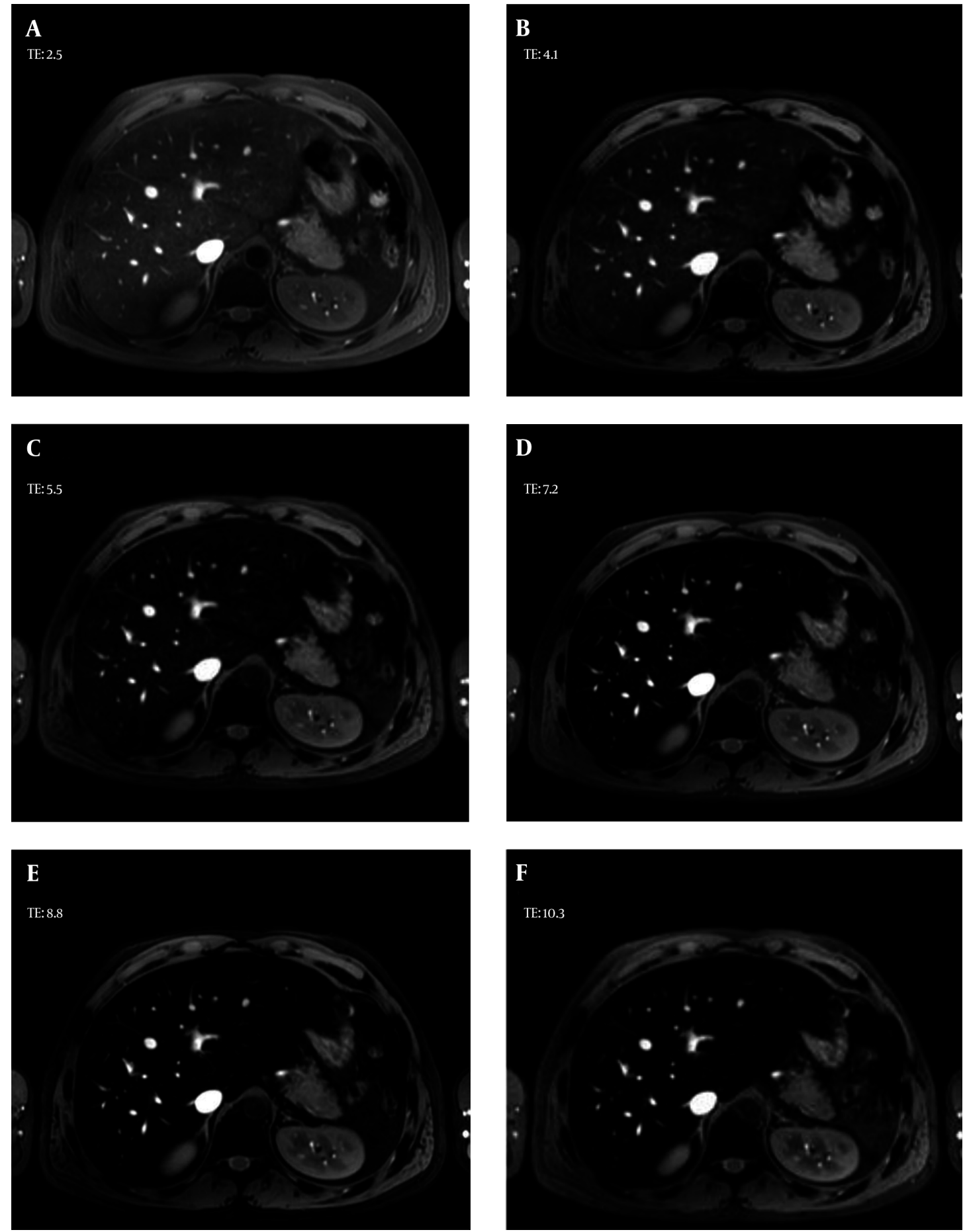

Figure 4. A-F, Multiecho fat supressed, gradient turbo field echo images in the axial plane at gradually increasing echo times passing through the level of the liver and both adrenals. There is severe hepatic iron deposition, seen as signal loss at the hepatic parenchyma as the echo time value increases. On the other hand, there is no significant signal drop in the adrenal glands in the same patient.

$\beta$-TM patients, most of the time, the biochemical adrenal insufficiency is not evident clinically with adrenal crisis. 
Symptoms that may be related to adrenal insuffiency such as chronic fatique, gastrointestinal problems and jointmuscle pain may also be encountered secondary to the presence of thalassemia. Diagnosis of adrenal insufficiency is critical because in stress conditions including surgery or sepsis, adrenal crisis may ensue. In addition, for $\beta$-TM patients with adrenal insufficiency, glucocorticoids are needed in the treatment of hypotenion accompanying cardiac insufficiency (16-18).

In thalassemia patients, in order to evalute the toxic level of iron, to plan chelation therapy and monitor the response to treatment, it is necessary to accurately detect the total iron load. Ferritin, which has been used widely for this purpose, is also well known as an acute phase reactant, whose levels may alter in inflammatory processes, malignancies, and liver diseases (19). It has been shown that liver iron load correlates with serum ferritin levels, but there is no good correlation between ferritin and iron load in the heart, pancreas, and hypophysis $(7,8,11)$. To evaluate liver and cardiac iron load, biopsy may be performed, but it is invasive and the complication rate of endomyocardial biopsy is rather high. The accuracy of biopsy is also limited due to uneven iron distribution in tissues. For the last two decades, non-invasive evaluation of iron overload with MRI has gained clinical acceptance, since it has been shown to be reproducible, and reliable. For the liver and heart, iron measurements have been performed in large patient groups with good histopathological correlation, and have proven to have high sensitivity and specificity values $(3,5-$ 7).

Intracellular ferritin and hemosiderin molecules that contain iron exert high paramagnetic properties, and their interaction with water molecules result in faster transverse magnetization (15). This in turn leads to signal loss, and tissues with high iron load are seen more hypointense. Iron accumulation is evaluated on MRI with two main techniques; namely, signal intensity ratio (SIR) and relaxometry. In the SIR technique, iron load is calculated by the ratio of target organ signal intensity to the signal intensity of a reference tissue that does not accumulate iron, such as the paraspinal muscles. This technique is known to have limitations. The most important is that saturation occurs in very high iron overload which prevents measurement of values above that range. Relaxometry technique calculates iron load by measuring the signal intensity loss values, which is induced by the paramagnetic properties of iron on different TEs) (20). Relaxometry methods can calculate $\mathrm{T} 2, \mathrm{~T} 2 *$ values after fitting decay models to the average signal intensity at different TEs. In this technique, signal intensity is plotted as a function of echo time that yields an automatic T2 parametric map. Although regarded as the most accurate technique, it also has some limitations that need to be overcome for standardized clinical utilization. The most important limitation of the relaxometry technique is the lack of concensus and presence of uncertainity about the accurate MRI acquisition parameters, which is currently overcome by obtaining a relatively large number of TEs and optimizing other sequence variables $(15,20)$.

As far as we could review the literature, there are very limited numbers of imaging studies related to iron deposition in the adrenal glands. Iron deposition in the adrenal glands could lead to adrenal insufficiency in stress conditions. Therefore, we believe that some $\beta$-TM patients will benefit from determination of iron deposition in the adrenal glands. In addition, accumulation of iron in the adrenal is also considered in the planning and response monitoring of chelation therapy. Long et al. (21) have evaluated iron overload in $\beta$-TM patients with computed tomography and reported the incidence of iron overload as 25.7\%, which presented as increased density. The only published study that evaluated iron load in the adrenal glands by MRI was performed retrospectively by Drakonaki et al. (3), They used SIR technique for hepatic iron quantification in a total of 35 thalassemia patients. In that study, to evaluate adrenal iron accumulation, a grading method was used from 0 to 2; 0 indicating normal adrenal gland signal intensity, 1 as hypointense on T2* only, and 2 as hypointense on at least $2^{*}$. According to their grading scale, $68 \%$ of the patient group had iron load evident with signal loss on the adrenal glands, when compared with the control group. In the same study, adrenal and hepatic iron load were found to be correlated. Whereas, no correlation was found between adrenal iron load and serum ferritin values (3). Their results are compatible with our study in terms of lack of correlation between adrenal iron deposition and serum ferritin values. On the other hand, in contrary to their data, in our study, there was no statistically significant correlation among iron deposition in the adrenal glands and liver. This difference could be attributed to various factors, such as possible difference in patient population, and more probably the difference in evaluation of adrenal iron deposition, which is rather semiquantitative in the former study, and quantitative in our study.

Performing iron quantification with 3 Tesla systems is challanging, partly because of increased magnetic inhomogeneity and specific absorbtion rate values. There are a limited number of studies that performed 3 Tesla for liver and cardiac iron measurements. They have proven that these measurements are feasible and reproducible in 3 Tesla (15). The major problem was reliable measurement of $2^{*}$ values in severe iron overload. The $\mathrm{T} 2{ }^{*}$ values tend to decrease in 3 Tesla systems when compared with 1.5 Tesla systems. Yet, there are no standardized criteria to grade iron accumulation in the liver and heart in 3 Tesla, which 
we believe needs to be established after studies including the high number of healthy and diseased individuals.

Our study has some limitations. The most important limitation is the lack of histopathological correlation although it is well known that MR has high sensitivity and specificity in the detection of iron overload in various organs. The other limitation is the relatively low number of patients.

In conclusion, adrenal iron load in $\beta$-TM patients can be reliably measured with relaxometry technique in 3 Tesla MRI. The results of this study highlight the absence of correlation between adrenal iron deposition both with serum ferritin values and hepatic iron load. A similar result has been shown in the literature previously, related with cardiac iron load. These variations among different organs might be related to the different iron uptake mechanisms or different response rates to chelator therapies. Further studies with higher numbers of patients are needed to both validate the efficiency of MRI in the detection of iron load, and to establish T2* decay cut-off values of the adrenal glands. We believe that this technique might also be used in the future to monitor the response to chelation therapy in other organs during the same MRI examination that also needs to be validated.

\section{Footnotes}

Authors' Contributions: Each author has equally contributed to this manuscript.

Financial Disclosure: Authors have no financial interests or conflicts.

\section{References}

1. Galanello R, Origa R. Beta-thalassemia. Orphanet J Rare Dis. 2010;5:11. doi: 10.1186/1750-1172-5-11. [PubMed: 20492708].

2. Weatherall DJ. The inherited diseases of hemoglobin are an emerging global health burden. Blood. 2010;115(22):4331-6. doi: 10.1182/blood2010-01-251348. [PubMed: 20233970].

3. Drakonaki E, Papakonstantinou O, Maris T, Vasiliadou A, Papadakis A, Gourtsoyiannis N. Adrenal glands in beta-thalassemia major: magnetic resonance (MR) imaging features and correlation with iron stores. Eur Radiol. 2005;15(12):2462-8. doi: 10.1007/s00330-005-2855-1. [PubMed: 16086182].

4. Rachmilewitz EA, Giardina PJ. How I treat thalassemia. Blood. 2011;118(13):3479-88. doi: 10.1182/blood-2010-08-300335. [PubMed: 21813448].

5. Angelucci E, Brittenham GM, McLaren CE, Ripalti M, Baronciani D, Giardini C, et al. Hepatic iron concentration and total body iron stores in thalassemia major. $N$ Engl J Med. 2000;343(5):327-31. doi: 10.1056/NEJM200008033430503. [PubMed: 10922422].

6. Tanner MA, Galanello R, Dessi C, Westwood MA, Smith GC, Nair SV, et al. Myocardial iron loading in patients with thalassemia major on deferoxamine chelation. J Cardiovasc Magn Reson. 2006;8(3):543-7. [PubMed: 16755844].
7. Kolnagou A, Economides C, Eracleous E, Kontoghiorghes GJ. Low serum ferritin levels are misleading for detecting cardiac iron overload and increase the risk of cardiomyopathy in thalassemia patients. The importance of cardiac iron overload monitoring using magnetic resonance imaging T2 and T2*. Hemoglobin. 2006;30(2):219-27. doi 10.1080/03630260600642542. [PubMed: 16798647].

8. Papakonstantinou O, Alexopoulou E, Economopoulos N, Benekos $\mathrm{O}$, Kattamis A, Kostaridou S, et al. Assessment of iron distribution between liver, spleen, pancreas, bone marrow, and myocardium by means of R2 relaxometry with MRI in patients with betathalassemia major. J Magn Reson Imaging. 2009;29(4):853-9. doi: 10.1002/jmri.21707. [PubMed: 19306409].

9. Wood JC, Enriquez C, Ghugre N, Tyzka JM, Carson S, Nelson MD, et al. MRI R2 and R2* mapping accurately estimates hepatic iron concentration in transfusion-dependent thalassemia and sickle cell disease patients. Blood. 2005;106(4):1460-5. doi: 10.1182/blood-2004-10-3982. [PubMed: 15860670].

10. Pennell DJ, Porter JB, Cappellini MD, Chan LL, El-Beshlawy A, Aydinok $\mathrm{Y}$, et al. Deferasirox for up to 3 years leads to continued improvement of myocardial T2* in patients with beta-thalassemia major. Haematologica. 2012;97(6):842-8. doi:10.3324/haematol.2011.049957. [PubMed: 22271905].

11. Papakonstantinou O, Ladis V, Kostaridou S, Maris T, Berdousi H, Kattamis $\mathrm{C}$, et al. The pancreas in beta-thalassemia major: MR imaging features and correlation with iron stores and glucose disturbances. Eur Radiol. 2007;17(6):1535-43. doi: 10.1007/s00330-006-05078. [PubMed: 17149622].

12. Papakonstantinou O, Drakonaki EE, Maris T, Vasiliadou A, Papadakis A, Gourtsoyiannis N. MR imaging of spleen in beta-thalassemia major. Abdom Imaging. 2015;40(7):2777-82. doi: 10.1007/s00261-015-04615. [PubMed: 26023008].

13. Sklar CA, Lew LQ, Yoon DJ, David R. Adrenal function in thalassemia major following long-term treatment with multiple transfusions and chelation therapy. Evidence for dissociation of cortisol and adrenal androgen secretion. Am J Dis Child. 1987;141(3):327-30. [PubMed: 3028128].

14. Vincent JM, Morrison ID, Armstrong P, Reznek RH. The size of normal adrenal glands on computed tomography. Clin Radiol. 1994;49(7):453-5. [PubMed: 8088036].

15. Storey P, Thompson AA, Carqueville CL, Wood JC, de Freitas RA, Rigsby CK. R2* imaging of transfusional iron burden at 3T and comparison with 1.5T. J Magn Reson Imaging. 2007;25(3):540-7. doi: 10.1002/jmri.20816. [PubMed: 17326089].

16. Macdonald RA, Mallory GK. Hemochromatosis and hemosiderosis Study of 211 autopsied cases. Arch Intern Med. 1960;105:686-700. [PubMed: 14419330].

17. Banani SA, Omrani GH. Cortisol and adrenocorticotropic hormone response to surgical stress (splenectomy) in thalassemic patients. Pediatr Surg Int. 2000;16(5-6):400-3. [PubMed: 10955573].

18. Huang KE, Mittelman SD, Coates TD, Geffner ME, Wood JC. A sig nificant proportion of thalassemia major patients have adrenal insufficiency detectable on provocative testing. J Pediatr Hematol Oncol. 2015;37(1):54-9. doi:10.1097/MPH.0000000000000199. [PubMed: 24942024].

19. Hoffbrand AV, Taher A, Cappellini MD. How I treat transfusional iron overload. Blood. 2012;120(18):3657-69. doi: 10.1182/blood-2012-05370098. [PubMed: 22919029].

20. Alustiza Echeverria JM, Castiella A, Emparanza JI. Quantification of iron concentration in the liver by MRI. Insights Imaging. 2012;3(2):17380. doi: 10.1007/s13244-011-0132-1. [PubMed: 22696043].

21. Long JJ, Doppman JL, Nienhus AW, Mills SR. Computed tomographic analysis of beta-thalassemic syndromes with hemochromatosis: pathologic findings with clinical and laboratory correlations. J Comput Assist Tomogr. 1980;4(2):159-65. [PubMed: 7365013]. 